\title{
Dermatofluoroscopy Is Also for Redheads a Sensitive Method of Early Melanoma Detection
}

\author{
Dieter Leupold ${ }^{a}$ Lukasz Szyc $^{\mathrm{b}}$ Goran Stankovic $^{\mathrm{b}}$ Maja Hofmann ${ }^{\mathrm{c}}$ \\ Matthias Scholz ${ }^{a}$ Andrea Forschner ${ }^{d}$ \\ a LTB Lasertechnik Berlin GmbH, Berlin, Germany; ${ }^{b}$ Magnosco GmbH, Berlin, Germany; ' University Department of \\ Dermatology, Charité Universitätsmedizin Berlin, Berlin, Germany; ${ }^{\mathrm{d} C e n t e r}$ of Dermatooncology, Department of \\ Dermatology, University Hospital Tübingen, Eberhard-Karls-University, Tübingen, Germany
}

\section{Keywords \\ Dermatofluoroscopy $\cdot$ Redheads $\cdot$ Melanoma}

\begin{abstract}
Background: Caucasians with red hair and fair skin have a remarkably increased risk of malignant melanoma compared to non-redhead Caucasians. Objectives: With the aim of a reliable melanoma diagnosis in redheads, the application of dermatofluoroscopy was analyzed in 16 patients with red hair. Most of them had been included in a clinical dermatofluoroscopy study for patients with the suspicion of melanoma. We examined whether the 25 lesions of the redheads showed the same characteristic melanin fluorescence spectra for dysplastic nevi and melanomas as those of non-redhead Caucasians or whether there was a different fluorescence pattern. This is important in view of the known significantly altered ratio of eumelanin to pheomelanin in the skin of redheads. Methods: More than 8,000 spatially resolved fluorescence spectra of 25 pigmented lesions were measured and analysed. The spectra were excited by the stepwise absorption of two $800-\mathrm{nm}$ photons (principle of dermatofluoroscopy). Furthermore, the fluorescence spectra of eumelanin and pheomelanin in hair samples were de-
\end{abstract}

termined in the same way. Results: The evaluation revealed that the melanin fluorescence spectra of dysplastic nevi and melanomas of redheads have the same spectral characteristics as those of non-redhead Caucasians. An accompanying result is that dermatofluoroscopy shows identical fluorescence spectra for eumelanin and pheomelanin. Conclusions: Dermatofluoroscopy proves to be a reliable diagnostic method also for redheads. Our results also explain our recent finding that there is a uniform fluorescence spectroscopic fingerprint for melanomas of all subtypes, which is of particular interest for hypomelanotic and apparently amelanotic melanomas containing pheomelanin.

๑) 2020 S. Karger AG, Basel

\section{Introduction}

Caucasians of red hair colour (RHC) - a phenotype characterized by red hair, fair skin, poor tanning, and often freckles - have an increased risk of melanoma compared to the normal Caucasian population with non-red hair [1-3]. The RHC phenotype is caused by loss of function mutations in the melanocortin receptor (MC1R) gene. Redheads primarily belong to Fitzpatrick skin type

\footnotetext{
Andrea Forschner

Center for Dermatooncology, Department of Dermatology University Hospital Tübingen

Liebermeisterstrasse 25, DE-72076 Tübingen (Germany)

andrea.forschner@med.uni-tuebingen.de
} 
Fig. 1. Flowchart of Patients and Methods. * For details of the FLIMMA study, see Forschner et al. [13].

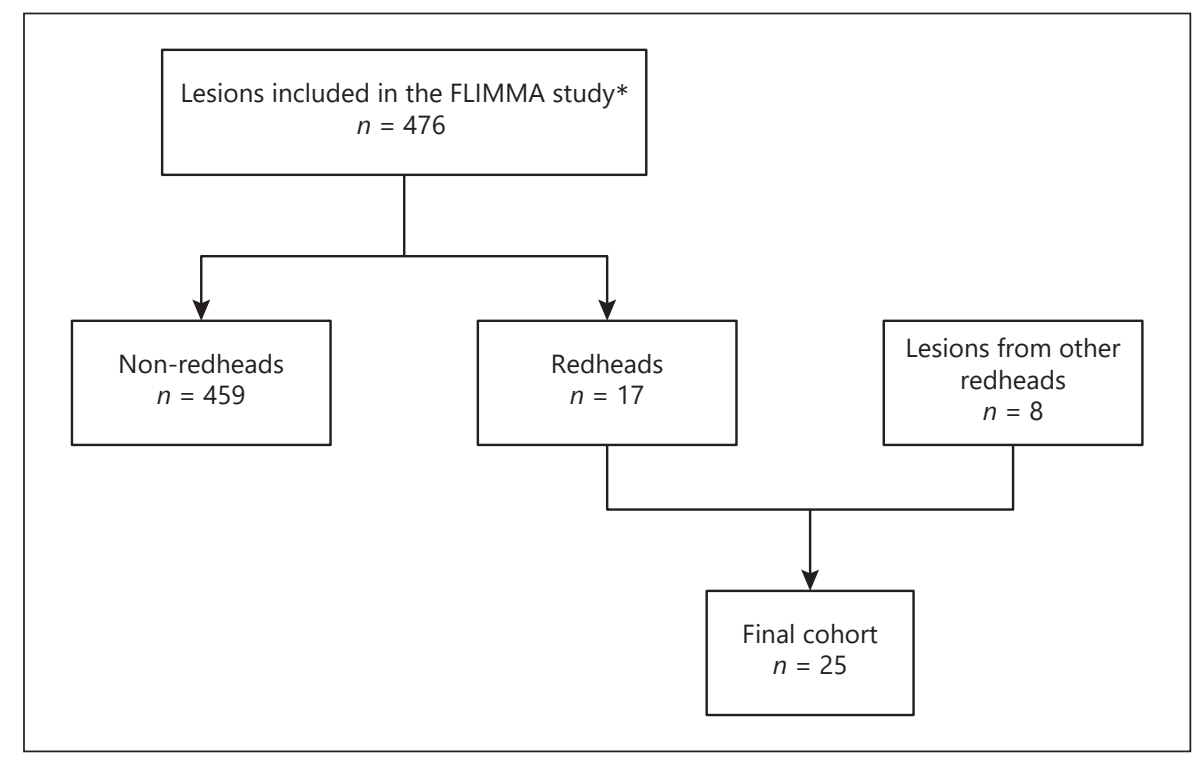

1 and about $75 \%$ of this skin type carries a dysfunctional $\mathrm{MC} 1 \mathrm{R}$ variant. This MC1R variant is found in only about $45 \%$ of skin type 2 and $<5 \%$ in higher skin types [4]. These $\mathrm{MC1R}$ variants harbour a significantly increased risk of developing cutaneous melanoma compared to wild-type patients. In the MC1R variant R160W (red hair and light skin), for example, the risk of melanoma is more than 8 times higher [2]. MC1R is a key regulator of skin pigmentation. The main effect of the loss of function mutation is a reduced melanin synthesis which is mainly at the expense of eumelanin, thus shifting the ratio of eumelanin/ pheomelanin to pheomelanin. This seems to be the cause of the increased melanoma risk of the RHC phenotype, as it reduces the more effective UV protection provided by eumelanin and promotes the two pheomelanin-based pathways for melanomagenesis: amplification of UV-induced reactive oxygen species and increased oxidative DNA damage [5]. Effective melanoma prevention is particularly important for persons of the RHC phenotype. In addition, a sensitive detection method for melanoma is crucial, preferably with special attention to the specific RHC pigmentation. Dermatofluoroscopy has recently proven to be a reliable diagnostic method for melanoma in Fitzpatrick skin types 2-4 [6-8]. The method is based on the two-photon excited fluorescence of melanin in the melanosomes of skin pigment cells. In this way, individual melanocytes, nevomelanocytes of benign and dysplastic nevi, and melanoma cells can be identified by four characteristic spectral profiles of their melanin fluorescence. For skin types 2-4 the ratio of eumelanin to pheomelanin increases from about 1 [9] in type 2 to values between 3 [10] and 21 [11] in type 4 . In particular, the variation of the eumelanin/pheomelanin ratio within these limits has no influence on the characteristic four fluorescence spectra of dermatofluoroscopy.

We investigated how dermatofluoroscopy works when we measure at a significantly lower ratio, as found in the RHC phenotype, where the eumelanin/pheomelanin ratio is below $1[9,12]$. The effect of such dominant pheomelanin content on the spectral profiles of dermatofluoroscopy has not yet been investigated. We performed these measurements on 21 pigmented lesions (nevi and melanomas) of redheads. In addition, 4 measurements on areas of normal pigmented skin of redheads were included. In total, more than 8,000 spectra were analysed. Accompanying fluorescence measurements were performed on red and black hair.

\section{Patients and Methods}

The present study included 14 redheads aged $23-73$ years ( 5 females and 9 males, 10 of skin type 1 and 4 of skin type 2). These redheads were participants in the clinical study FLIMMA [13, 14], with a total of 17 suspicious atypical lesions (Fig. 1), intended for excision and mainly localized on arms and legs, back, and lower abdomen. Prior to excision, the lesions were examined by dermatofluoroscopy with the Derma FC device (see method). Subsequently, the excised specimens were histologically evaluated. This revealed 9 dysplastic nevi, 3 melanomas (all superficial spreading melanomas) and 1 seborrheic keratosis in skin type 1 , and 2 dysplastic nevi and 2 melanomas (melanoma in situ and superficial spreading melanomas) in skin type 2 . The proportion of 5 melanomas among 17 clinically suspicious lesions (29.4\%) in redheads 


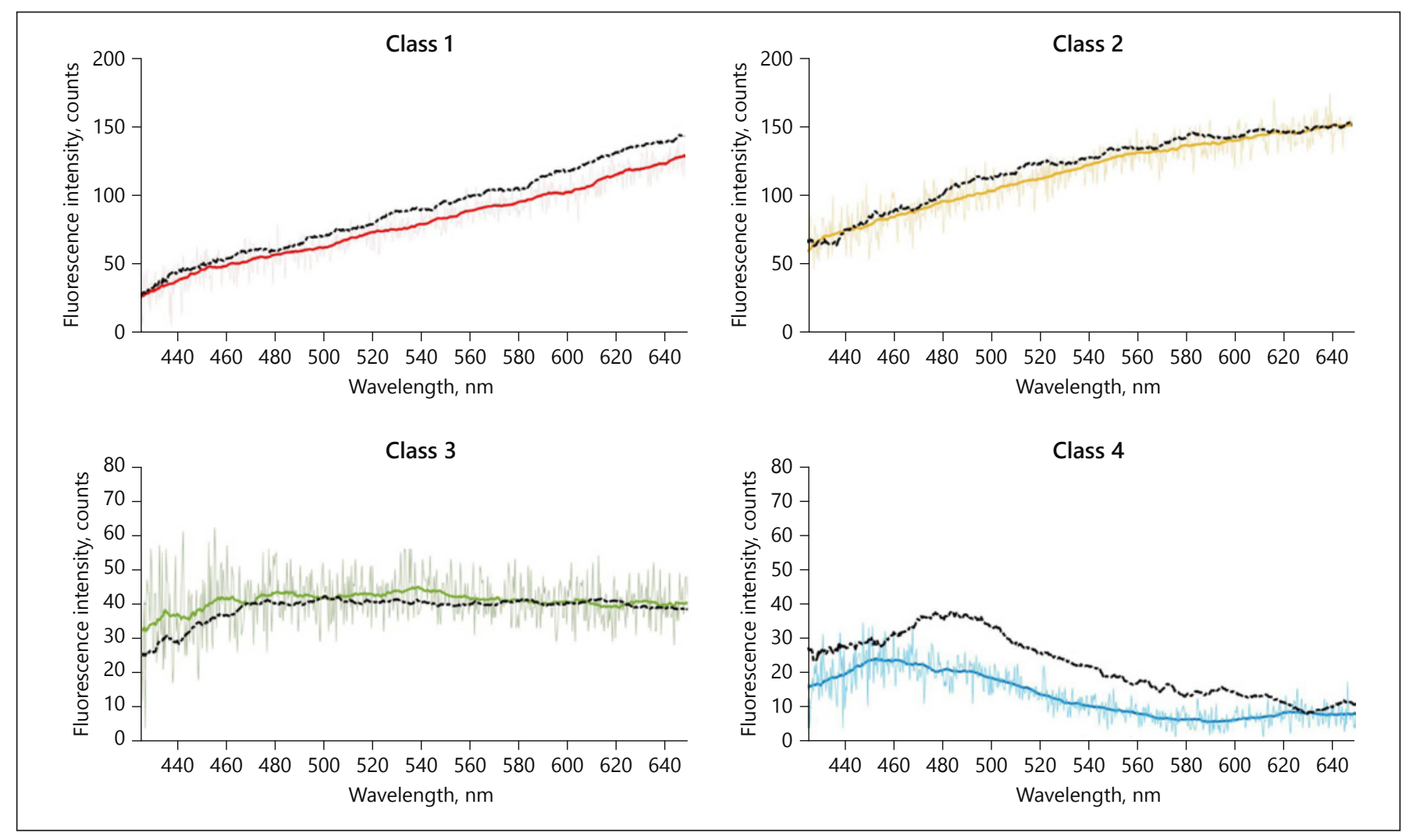

Fig. 2. Dermatofluoroscopy of skin tissue from Caucasians (Fitzpatrick types 2-4) in vivo. The four representative classes of melanin-dominated spectra - upper left: class 1, melanoma; upper right: class 2, dysplastic nevus; lower left: class 3, benign nevus; lower right: class 4, normal pigmented skin. The dashed black line shows in each case the characteristic spectral shape in non-redhead

is higher than the proportion of 95 melanomas among 457 suspicious lesions $(20.8 \%)$ in all other non-RHCs of the clinical study FLIMMA.

In addition to the 14 redheads in the FLIMMA study, 8 lesions from 2 redheads (both female; aged 22 and 23 years) who voluntarily participated in Derma FC examinations were added to expand the fluorescence database. Written consent was obtained from these additional participants for testing with Derma FC and also for publication of the results. These 8 lesions consisted of 4 clinically benign nevi and 4 areas of normally pigmented skin of the arms. These 8 samples were not excised.

The experimental basis for the investigation of cutaneous pigmentation is dermatofluoroscopy, a new method of selectively excited fluorescence of melanin [6-8]. This melanin fluorescence of the melanosomes is ultra-weak and is completely masked by the much more intense fluorescence of other endogenous fluorophores, e.g., $\mathrm{NAD}(\mathrm{P}) \mathrm{H}$ and flavins (the so-called autofluorescence of the skin), when using conventional single-photon excitation.

The key of dermatofluoroscopy is the stepwise approach of two-photon excitation of melanin with nanosecond pulses at 800 $\mathrm{nm}$. This type of excitation is unique for melanin compared to all other endogenous fluorophores such as $\mathrm{NADH}$ and flavins. The

Caucasians for the individual classes. The coloured lines show characteristic examples of spectral shapes of redhead Caucasians. For class 4 of the redheads, additionally an example of low pigmentation demonstrates the hypsochromic shift compared to the standard spectrum (reduced melanin fluorescence compared to the generally slight autofluorescence).

melanin fluorescence detectable in this way is measured in the spectral range between 400 and $650 \mathrm{~nm}$. It has a high information content on structural and morphological questions, which are reflected in the different spectral forms: occurrence of nevomelanocytes in nests and in pronounced nevi, indication of incipient melanomagenesis, as well as occurrence of pronounced melanomas. The melanocytes also show a typical melanin spectrum, with a small contribution of autofluorescence. The melanin fluorescence spectra also provide information about quantitative changes in the melanin concentration, which are reflected in the fluorescence intensity.

To date, several hundred Caucasians have been examined by dermoscopy for normal pigmented skin, benign and dysplastic nevi, and melanomas. This results in a database of several tens of thousands of spectra, the vast majority of which can be assigned to one of the following four classes of melanin-dominated fluorescence spectra by means of automated spectral identification with fixed root mean squared error: melanosomes of melanocytes (Fig. 2, class 4, black curve), melanosomes of nevomelanocytes of benign nevi (Fig. 2, class 3, black curve), melanosomes of nevomelanocytes of dysplastic nevi (Fig. 2, class 2, black curve), and melanosomes of melanoma cells (Fig. 2, class 1, black curve). The spec- 


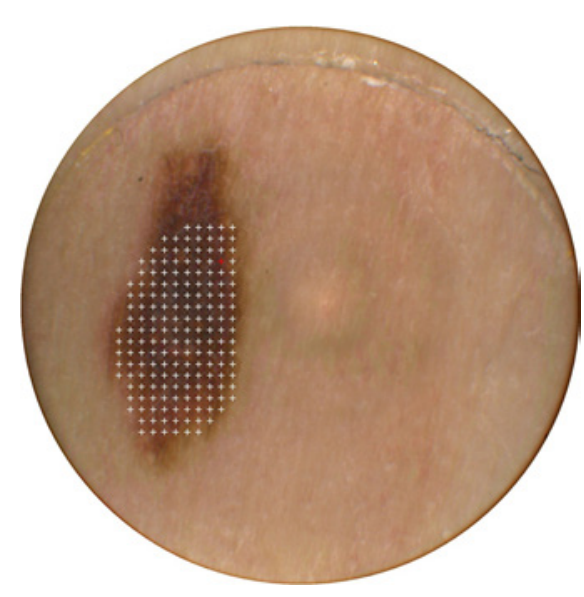

Fig. 3. Dermatofluoroscopy of a dysplastic nevus from a redhead; the grid pitch is $200 \mu \mathrm{m}$. The crosses mark the locations of the fluorescence measurements. White crosses mark healthy micro-areas (spectra of classes 3 or 4). The pink crosses mark micro-areas with incipient malignant melanocytic degeneration (class 2), and the red crosses mark micro-areas with fluorescence typical for melanoma cells (class 1). In this figure there is only one single red cross.

tral course of the latter shows a constant increase in intensity from 440 to $650 \mathrm{~nm}$ (mathematically described, this means that the first derivative of the spectral fluorescence course $\operatorname{dIF}(\lambda) / \mathrm{d} \lambda$ is a horizontal straight line between 440 and $650 \mathrm{~nm}$ ). This is a characteristic of all melanoma subtypes investigated so far, such as superficial spreading melanoma, lentigo maligna melanoma, nodular melanoma, and melanoma in situ [15]. The spectral fluorescence course of dysplastic nevi (Fig. 2, class 2) differs from the course according to class 1 by a reduced increase above about $570 \mathrm{~nm}$ (which means a decrease of the constant value of the first derivative above $570 \mathrm{~nm}$ ). The melanin fluorescence spectra of melanocytes (Fig. 2, class 4) show a more distinct band peak at about $490 \mathrm{~nm}$ (i.e., a zero crossing of the first derivative at about $490 \mathrm{~nm}$ ), and benign nevi show a flat band as shown in Figure 2, class 3 (with a zero crossing of the first derivative in the range between about 530 and $550 \mathrm{~nm})$.

Spectra that do not fit into this classification of cutaneous melanin spectra, e.g., from hair, marker fluorophores, or impurities, are either assigned to other classified spectra or marked as unidentifiable. The latter could apply to the fluorescence spectra of the redheads if they differ significantly from those of normal Caucasians.

Dermatofluoroscopy is performed with the Derma FC device (Magnosco GmbH, Berlin, Germany). The Derma FC is a CE-certified Class IIa medical device approved for the European market. This certificate is based on a prospective multicentre clinical study with 620 suspected melanoma lesions [13]. The skin area of interest is covered with a measuring grid. The grid spacing is $200 \mu \mathrm{m}$; the diameter of each skin area analysed spectroscopically is $30 \mu \mathrm{m}$. In this way, typically several hundred fluorescence spectra are obtained per skin area examined. For more detailed technical information, please refer to Forschner et al. [13].

Dermatofluoroscopy in Redheads

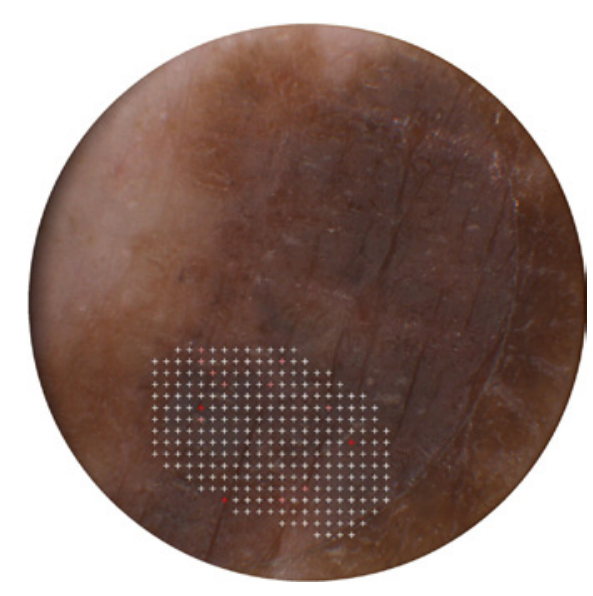

Fig. 4. Dermatofluoroscopy of a melanoma from a redhead, the grid pitch is $200 \mu \mathrm{m}$. The crosses mark the locations of the fluorescence measurements. White crosses mark healthy micro-areas (spectra of classes 3 or 4). The pink crosses mark micro-areas with incipient malignant melanocytic degeneration (class 2), and the red crosses mark micro-areas with fluorescence typical for melanoma cells (class 1$)$.

Dermatofluoroscopy was used to measure 25 skin areas in vivo of redheads, including 17 clinically suspicious pigmented lesions, which were identified after excision in 5 cases as melanoma, in 11 cases as dysplastic nevi, and in 1 case as seborrheic keratosis. In addition, 4 clinically benign nevi and 4 areas of normal pigmented skin were measured. For each skin area the measurement grid included several hundred measurement points (for examples of measurement grids, see Fig. 3, 4), so that a total of more than 8,000 melanin fluorescence spectra of the skin of redheads were obtained.

\section{Results}

The vast majority of the measured skin spectra of lesions $(8,467$ of 8,534$)$ show the same spectral characteristics as the standard spectra of non-red Caucasians and thus follow the same classification (classes 1-4). This applies to the melanin fluorescence of melanocytes as well as that of benign and dysplastic nevomelanocytes and of melanoma cells. To illustrate this behaviour, Figure 2 shows representative spectra of redheads of the four classes (the coloured curves in Fig. 2) in addition to the standard spectra of normal Caucasians. With particularly low pigmentation, there is a slight modification in class 4, which is a superposition of the melanin fluorescence of the melanosomes with a low amount of autofluorescence (melanin fluorescence maximum at about $490 \mathrm{~nm}$, autofluorescence at about $460 \mathrm{~nm}$ ). There is a hypsochromic/ 
Fig. 5. Difference spectra of black hair (a) and red hair (b) minus hair spectra of the oculo-cutaneous albino. The difference spectra represent dominantly eumelanin (a) and pheomelanin (b). The 14 columns each represent spectrally integrated averaged intensity values from measurements on 10 hair samples each of the type described, each with an average SD of $15 \%$. The spectra were measured with a predecessor variant of DERMA FC, the LIMES $16 \mathrm{P}[20]$. The main difference between the two devices is the use of a 16-channel photomultiplier in the LIMES $16 \mathrm{P}$ instead of a CCD camera in the DERMA FC.

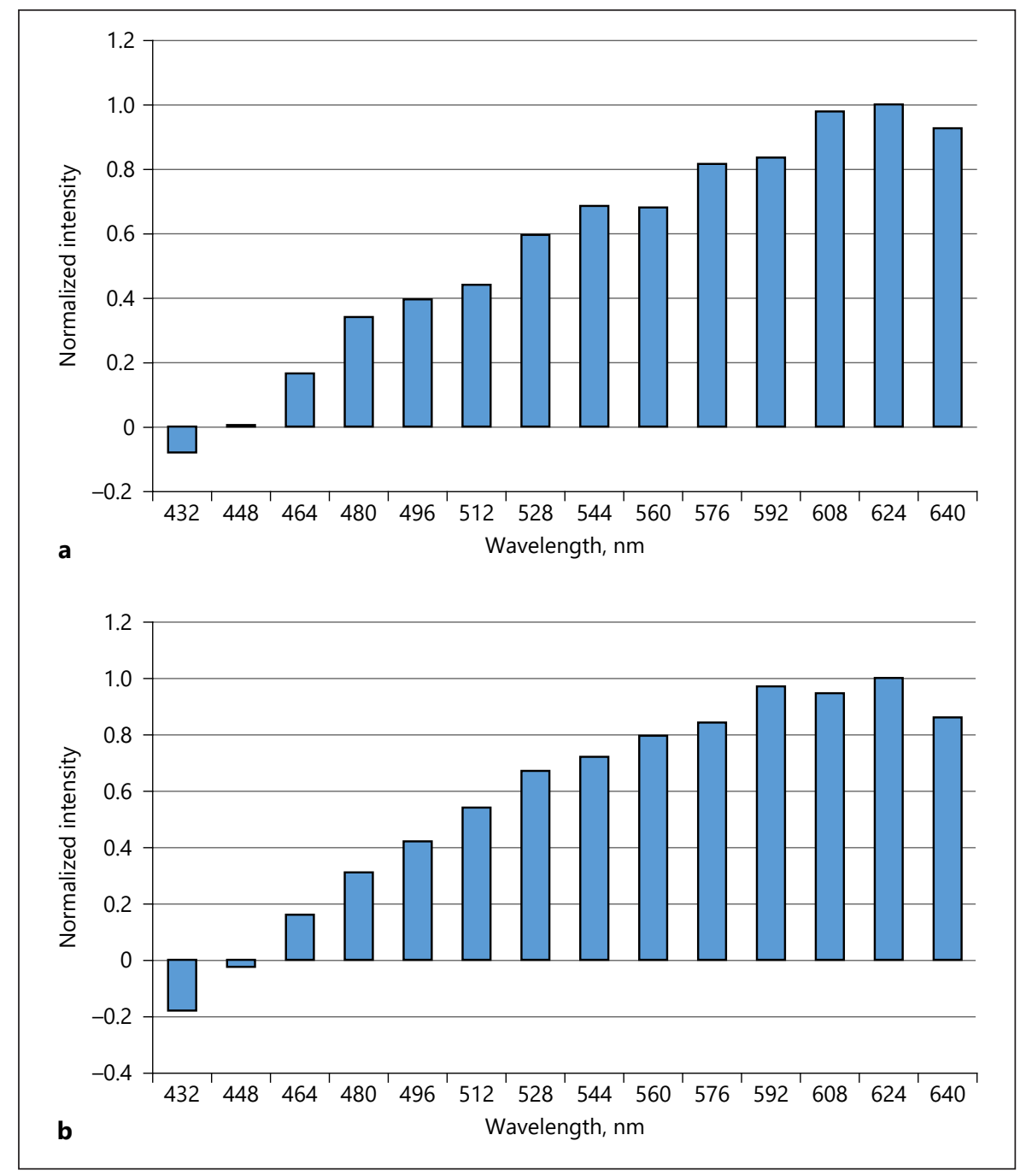

blue shift of the band maximum due to a reduced contribution of melanin fluorescence. This shift is also shown in Figure 2.

Since the fluorescence spectroscopic results of redheads correspond to those of non-redhead Caucasians, the dermatofluoroscopic examination of suspicious pigmented lesions is also suitable for redheads and is a safe method for the early detection of melanoma in this highrisk melanoma cohort [13]. This is reflected in the results obtained with Derma FC on the 17 clinically suspect pigmented lesions considered here: of the 5 histologically identified melanomas, 4 were classified as melanomas by Derma FC (sensitivity $=80 \%$ ). Of the 12 non-melanomas (11 dysplastic nevi, 1 seborrheic keratosis), 6 were identified as such (specificity $=50 \%$ ). Taking into account the small number of cases for redheads, these values match the corresponding values of the overall study [13] (specificity $=89.1 \%$; sensitivity $=44.8 \%$ ). This means that the automatic evaluation of the DERMA FC device can also be applied to redheads. Examples of a measurement grid on a dysplastic nevus and melanoma (superficial spreading melanomas) of redheads in vivo are shown in Figures 3 and 4 . Both diagnoses were made after excision by dermatohistologists. The crosses mark the locations of the fluorescence measurements/evaluation. White crosses mark healthy micro-areas (class 3 or 4 spectra in Fig. 2). The pink crosses mark micro-regions with incipient malignant melanocytic degeneration (class 2 in Fig. 2) and the red crosses mark micro-regions with fluorescence typical of melanoma cells (class 1 in Fig. 2). With only one micro-region with a class 1 spectrum, the nevus shown in Figure 3 is considered benign in the clinical sense. The 
Fig. 6. Energy level scheme (Jablonski scheme for organic molecules with pi-electron systems, adapted to melanin in vivo) to illustrate the dermatofluoroscopy relevant excitation and relaxation processes in melanin configurations in the melanosomes of nevi $(\mathrm{N})$ and melanomas $(\mathrm{M})-1$ : ground state; 2: excited state, reached after the first absorption; $2(\mathrm{~N})$ : relaxed state 2 in nevi, initial state of second absorption; 2(M): the corresponding in melanomas; 3(N), 3(M): excited state, reached after the second absorption in nevi or melanomas. Rightward arrows: absorption; leftward arrows: fluorescence; wavy arrows $k(\mathrm{~N})$ or $k(\mathrm{M})$ : relaxation processes in nevi or in melanomas.

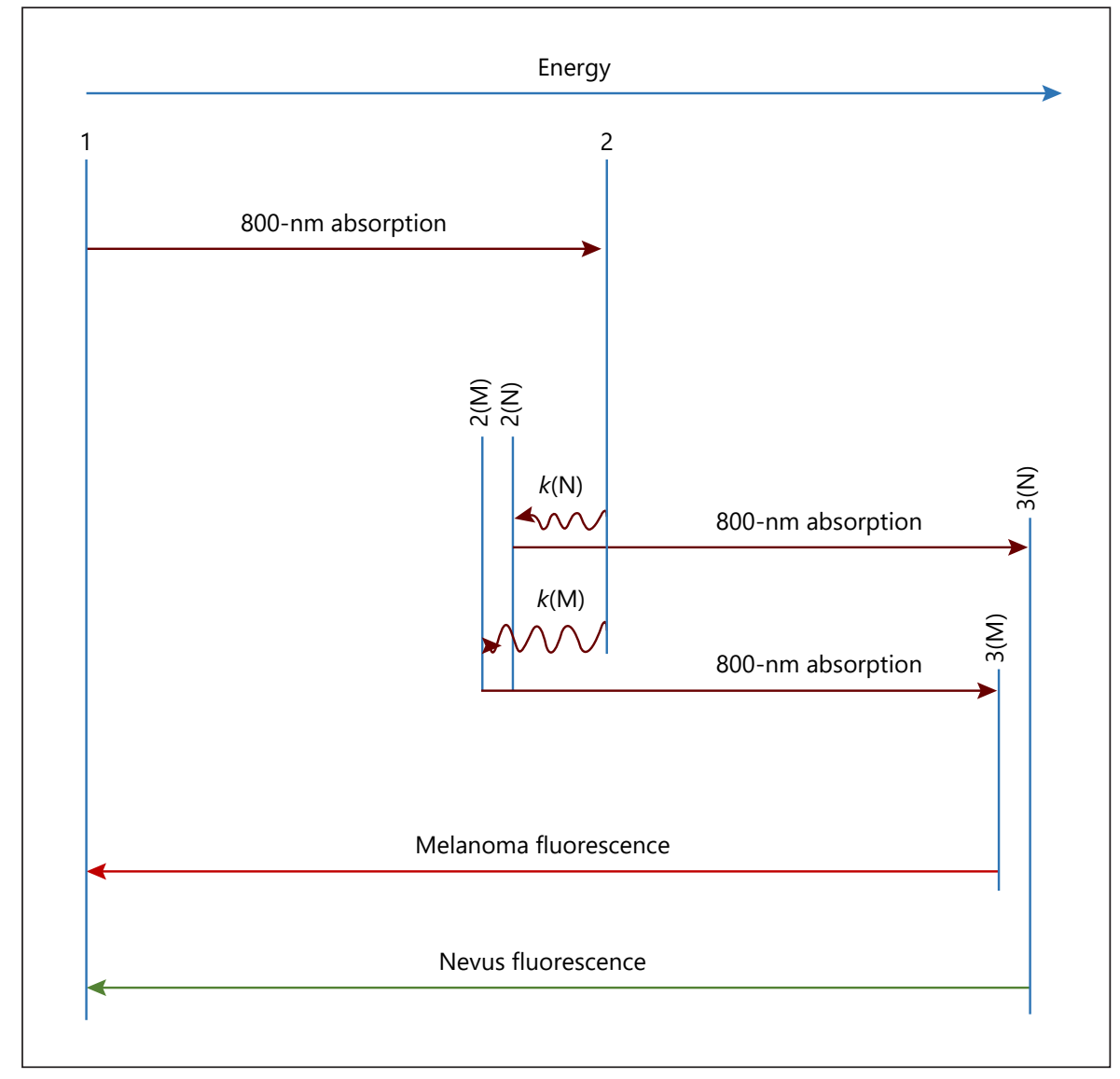

resulting score from Derma FC is well below the classification as a dysplastic nevus. The measurement grid of a melanoma presented in Figure 4 shows 10 micro-areas of class 2 and 3 micro-areas of class $1 ; 4$ of these class 2 areas are connected (clusters), and another cluster consists of one class 1 and one class 2 . The corresponding score from Derma FC clearly indicates a melanoma.

Since the melanin spectra from the melanosomes of cutaneous melanocytes (class 4 ) of redheads and normal Caucasians of all Fitzpatrick types up to type 4 are based on a wide variation range of the ratio of eumelanin to pheomelanin (from $<1$ up to $>>1$ ), the indistinguishability of their melanin fluorescence spectra means that under the given excitation conditions (stepwise two $800-\mathrm{nm}$ photon absorption) the fluorescence spectra of eumelanin and pheomelanin in the cutaneous melanocytes are identical.

This conclusion is supported by the melanin spectra of the hair. The evidence for the relationship of eumelanin and pheomelanin in hair is very different $[16,17]$. The greatest difference in this ratio is found between red and black hair: dominant eumelanin in black hair, but in red hair about 1:1 or dominant pheomelanin.

Dermatofluoroscopy in Redheads
When black and red hair samples are excited in the same way as the cutaneous melanin spectra, i.e., by stepwise absorption of two 800 -nm photons, they show identical fluorescence spectra. The fluorescence of hair components other than melanin is detected by measurement on hair from an oculocutaneous albino and eliminated from the red and black hair spectra to determine the pure melanin spectra. These different spectra are shown in Figure 5. The apparent identity of the melanin spectra of red and black hair proves that eumelanin and pheomelanin have identical spectral properties in hair.

\section{Discussion}

It is known that the ratio of eumelanin to pheomelanin varies significantly: (i) between skin types 1-4 [9-12], (ii) within the progression from benign nevi to melanoma (class $3->$ class $2->$ class 1 ) $[18,19]$, and (iii) between the different melanoma subtypes $[8,16]$. Remarkably, the results described here show that all 4 classes of melanin fluorescence spectra in vivo (melanocytes, benign and dys- 
plastic nevi, melanomas; Fig. 2) are independent of this eumelanin/pheomelanin ratio. This independence is particularly important for melanomas. Based on experimental results on in situ melanomas, superficial spreading melanoma, nodular melanoma, and lentigo maligna melanoma, we have already described in previous work [8] a spectral behaviour-like class 1 as a global fingerprint of the melanoma, independent of the subtype. This was confirmed in all further measurements, also in ocular melanoma [15]. This means that the melanin configuration responsible for the melanoma fluorescence fingerprint is the same for all melanoma subtypes, but in this configuration the eumelanin/pheomelanin ratio can vary without affecting the fingerprint.

Given the independence of the spectral course of melanin fluorescence from the eumelanin/pheomelanin ratio, it is necessary to ask how the fluorescence redshift between nevi and melanoma can be explained. The basis for the understanding is illustrated in Figure 6 in the Jablonski term scheme for organic molecules with pielectron systems, adapted to melanin in vivo:

(a) The spectral position of the melanin fluorescence is determined by the energy difference between the excited state reached after stepwise two photon absorption (step 3 for nevi (N), step 3 for melanomas (M)) and the ground state (step 1) (for clarification, we generally restrict ourselves in the schematic representation to "nevi" without differentiating between benign and dysplastic).

(b) The energy of this excited state $3(\mathrm{~N})$ or $3(\mathrm{M})$ (the initial level of fluorescence) is mainly determined by the relaxation behaviour of melanin in excited state 2 . This is achieved by the absorption of a first $800-\mathrm{nm}$ photon. There the melanin - still in the nuclear configuration of the ground state - meets the electron distribution of this excited state. This changes immediately at the $800-\mathrm{nm}$ electron transition. The relaxation $(k(\mathrm{~N})$ or $k(\mathrm{M}))$ is now the adjustment of the nuclear configuration to the electron configuration of this excited state. Afterwards, the thermal relaxation begins, which is very effective for melanin. This energetically downwards-directed process is stopped as soon as the strength of the second $800-\mathrm{nm}$ absorption (energy upwards) exceeds the downwards-directed relaxation. Both the rate of energy dissipation and the strength of the second absorption can be different in nevi and melanoma. Accordingly, the initial state of fluorescence transition is energetically lowered in different ways and the fluorescence is redshifted in different ways.

(c) Both competing processes are determined by the melanin configuration, which results from the polymerization of the building blocks DHI and DHICA to eu- melanin, benzthiazole, and benzthiazine to pheomelanin - their cross-linking by carbon bonds and the formation of stacked structures by pi-electronic interactions in the melanosomes of the melanoma cells and nevomelanocytes [23]. Both processes are also influenced by the immediate melanin micro-environment, the way in which the melanin chemically binds to the structural proteins of the scaffold (matrix of fibrils).

It is important to note that further interactions, such as those between melanosomes and keratinocytes, do not affect the melanin fluorescence spectra described here, i.e., they do not influence the competing processes described above. This important fact results from the experimental finding that the melanin fluorescence spectra of types 1-4 are largely identical in vivo (intact skin), in vitro (excised specimen), and in histological FFPE preparation $[7,8,15,20]$. This means that it is irrelevant for melanoma fluorescence fingerprinting whether the melanosomes are partially broken in melanoma cells.

The uniform melanin fluorescence of all main melanoma subtypes investigated so far (melanoma fingerprint) therefore means that their melanin configuration is identical - only the "population" with eumelanin or pheomelanin at the individual points of the configuration may vary.

This uniform melanin configuration is also independent of the genetic changes in the subtypes [21]. This native melanin configuration, i.e., the structure of the epidermal melanin grains (30- to $50-\mathrm{nm}$ nanoparticles) is not yet known [12]; the current state of knowledge is presented in D'Alba et al. [22] and Micillo et al. [23]. Current theoretical studies on the derivation of absorption spectra and their possible substructures from structural models are presented in Micillo et al. [23] and Ju et al. [24]. This applies analogously to the melanin configuration (different from melanoma) in the melanosomes of benign or dysplastic nevi.

It should be added that our original hypothesis to explain the redshift between nevi and melanoma [8] was based on the faster relaxation of pheomelanin in the excited state compared to eumelanin. This is now obsolete.

The studies described above all relate to Fitzpatrick types 1-4, concerning Fitzpatrick types 5 and 6 , it has to be considered that the ratio of eumelanin to pheomelanin is further increased [25]. Our first dermatofluoroscopic investigations on a patient with skin type 5 with benign nevi of the compound and congenital type [15] show a different fluorescence behaviour than that in types 1-4, e.g., the spectra of benign nevi (class 3 ) are redshifted compared to those of class 3 in Fitzpatrick types 1-4. Possible causes may be as- 
sociated with an increased DHI to DHICH eumelanin ratio [24] and possibly a resulting altered native melanin configuration. A different melanin behaviour is also documented, e.g., in the different depositions of melanin granules on the nuclei of keratinocytes (aggregated vs. monomeric). It agrees with the above-mentioned that Derma-FC is not (yet) approved for skin types 5 and 6 .

\section{Conclusion}

Since pheomelanin and eumelanin show identical fluorescence spectra under the special excitation conditions of dermatofluoroscopy, the melanosomes of dysplastic nevomelanocytes and melanoma cells of redheads show the same characteristic melanin fluorescence spectra as those of normal Caucasians. Dermatofluoroscopy is therefore also a reliable melanoma diagnosis for redheads. It is also suitable for pheomelanin-dominated hypomelanotic and apparently amelanotic melanomas, which often contain pheomelanin [26]. The recognition and diagnosis of these melanomas is otherwise a challenge $[27,28]$. Overall, the identification of the class 1 spectral fluorescence profile was verified as a uniform spectral fingerprint of melanomas in skin types 1-4, which also qualifies dermatofluoroscopy in redheads for early diagnosis of melanoma.

\section{Key Message}

This is the first dermatofluoroscopic analysis with focus on redheads, demonstrating its reliability of early melanoma detection.

\section{Acknowledgements}

We thank the whole study team for collecting the data and the patients for their participation.

\section{Statement of Ethics}

The research complies with the guidelines for human studies and was carried out in accordance with the World Medical Association Declaration of Helsinki.

\section{Disclosure Statement}

M.S. is the Managing Director of LTB Lasertechnik Berlin $\mathrm{GmbH}$, which developed the dermatofluoroscopy technique. L.S. is Head of Science and G.S. is Head of Development in Magnosco $\mathrm{GmbH}$, Berlin. No conflicts of interest were declared by D.L., M.H., and A.F.

\section{Funding Sources}

The University of Tübingen acted as the legal sponsor of the study. The study was funded by Magnosco GmbH (c/o LTB Lasertechnik Berlin GmbH, Am Studio 2c, 12489 Berlin).

\section{Author Contributions}

Conception: D.L., A.F., and M.S. Investigations/experimental section: A.F., M.H., and G.S. Data evaluation: D.L., A.F., L.S., and M.S. Draft of the manuscript: D.L., A.F., and L.S. Final version of the manuscript: all authors.

\section{References}

1 Duffy DL, Lee KJ, Jagirdar K, Pflugfelder A, Stark MS, McMeniman EK, et al. High naevus count and MC1R red hair alleles contribute synergistically to increased melanoma risk. $\mathrm{Br}$ J Dermatol. 2019 Nov;181(5):1009-16.

2 Pasquali E, García-Borrón JC, Fargnoli MC, Gandini S, Maisonneuve P, Bagnardi V, et al.; M-SKIP Study Group. MC1R variants increased the risk of sporadic cutaneous melanoma in darker-pigmented Caucasians: a pooled-analysis from the M-SKIP project. Int J Cancer. 2015 Feb;136(3):618-31.

3 Raimondi S, Sera F, Gandini S, Iodice S, Caini S, Maisonneuve $\mathrm{P}$, et al. MC1R variants, melanoma and red hair color phenotype: a meta-analysis. Int J Cancer. 2008 Jun;122(12):2753-60.

4 Valverde P, Healy E, Jackson I, Rees JL, Thody AJ. Variants of the melanocyte-stimulating hormone receptor gene are associated with red hair and fair skin in humans. Nat Genet. 1995 Nov;11(3):328-30.
5 Mitra D, Luo X, Morgan A, Wang J, Hoang MP, Lo J, et al. An ultraviolet-radiation-independent pathway to melanoma carcinogenesis in the red hair/fair skin background. Nature. 2012 Nov;491(7424):449-53.

6 Leupold D, Giering HG. Dermatofluoroscopy. In: Stolz W, Hänßle H, Sattler E, Weber J, editors. Bildgebende Diagnostik in der Dermatologie. Stuttgart/New York: Georg Thieme Verlag; 2018. p. 263-65.

7 Scholz M, Stankovic G, Scholz C, Leupold D, Buder S, Kohl P, et al. En route to a new in vivo diagnostic of malignant pigmented melanoma. Pigment Cell Melanoma Res. 2012 Mar; 25(2):281-3.

8 Leupold D, Scholz M, Stankovic G, Reda J, Buder S, Eichhorn R, et al. The stepwise two-photon excited melanin fluorescence is a unique diagnostic tool for the detection of malignant transformation in melanocytes. Pigment Cell Melanoma Res. 2011 Jun;24(3):438-45.
9 Krasieva TB, Stringari C, Liu F, Sun CH, Kong Y, Balu M, et al. Two-photon excited fluorescence lifetime imaging and spectroscopy of melanins in vitro and in vivo. J Biomed Opt. 2013 Mar; 18(3):31107.

10 Del Bino S, Ito S, Sok J, Nakanishi Y, Bastien $\mathrm{P}$, Wakamatsu K, et al. Chemical analysis of constitutive pigmentation of human epidermis reveals constant eumelanin to pheomelanin ratio. Pigment Cell Melanoma Res. 2015 Nov;28(6):707-17.

11 Hani AF, Baba R, Shamsuddin N, Nugroho $\mathrm{H}$. Determination of melanin types and relative concentrations: an observational study using a non-invasive inverse skin reflectance analysis. Int J Cosmet Sci. 2014 Oct;36(5): 451-8.

12 Nasti TH, Timares L. MC1R, eumelanin and pheomelanin: their role in determining the susceptibility to skin cancer. Photochem Photobiol. 2015 Jan-Feb;91(1):188-200. 
13 Forschner A, Keim U, Hofmann M, Spänkuch I, Lomberg D, Weide B, et al. Diagnostic accuracy of dermatofluoroscopy in cutaneous melanoma detection: results of a prospective multicentre clinical study in 476 pigmented lesions. Br J Dermatol. 2018 Aug;179(2):47885.

14 Fink C, Hofmann M, Jagoda A, Spaenkuch I, Forschner A, Tampouri I, et al. Study protocol for a prospective, non-controlled, multicentre clinical study to evaluate the diagnostic accuracy of a stepwise two-photon excited melanin fluorescence in pigmented lesions suspicious for melanoma (FLIMMA study). BMJ Open. 2016 Dec;6(12):e012730.

15 Leupold D, Scholz M, Stankovic G, Pfeifer L, Giering HG, Buder S, et al. Uniform spectral fingerprint of the different melanoma subtypes: diagnostic utility and mechanistic implications. 11th International Congress of the Society for Melanoma Research, Wien. Pigment Cell Melanoma Res. 2014;27:S6.

16 Ito S, Wakamatsu K. Quantitative analysis of eumelanin and pheomelanin in humans, mice, and other animals: a comparative review. Pigment Cell Res. 2003 Oct;16(5):523-31.
17 Ito S, Wakamatsu K. Diversity of human hair pigmentation as studied by chemical analysis of eumelanin and pheomelanin. J Eur Acad Dermatol Venereol. 2011 Dec;25(12):1369-80.

18 Pavel S, van Nieuwpoort F, van der Meulen $\mathrm{H}$, Out C, Pizinger K, Cetkovska P, et al. Disturbed melanin synthesis and chronic oxidative stress in dysplastic naevi. Eur J Cancer. 2004;40(9):1423-30.

19 Marchesini R, Bono A, Carrara M. In vivo characterization of melanin in melanocytic lesions: spectroscopic study on 1671 pigmented skin lesions. J Biomed Opt. 2009 Jan-Feb; 14(1):014027

20 Eichhorn R, Wessler G, Scholz M, Leupold D, Stankovic G, Buder S, et al. Early diagnosis of melanotic melanoma based on laser-induced melanin fluorescence. J Biomed Opt. 2009 May-Jun;14(3):034033.

21 Shain AH, Yeh I, Kovalyshyn I, Sriharan A, Talevich E, Gagnon A, et al. The genetic evolution of melanoma from precursor lesions. $\mathrm{N}$ Engl J Med. 2015 Nov;373(20):1926-36.

22 D’Alba L, Shawkey MD. Melanosomes: Biogenesis, Properties, and Evolution of an Ancient Organelle. Physiol Rev. 2019 Jan;99(1):1-19.
23 Micillo R, Panzella L, Iacomino M, Prampolini G, Cacelli I, Ferretti A, et al. Eumelanin broadband absorption develops from aggregation-modulated chromophore interactions under structural and redox control. Sci Rep. 2017 Feb;7(1):41532.

24 Ju KY, Fischer MC, Warren WS. Understanding the role of aggregation in the broad absorption bands of eumelanin. ACS Nano. 2018 Dec;12(12):12050-61.

25 Alaluf S, Heath A, Carter N, Atkins D, Mahalingam $\mathrm{H}$, Barrett $\mathrm{K}$, et al. Variation in melanin content and composition in type V and VI photoexposed and photoprotected human skin: the dominant role of DHI. Pigment Cell Res. 2001 Oct;14(5):337-47.

26 Wang $\mathrm{H}$, Osseiran S, Igras V, Nichols AJ, Roider EM, Pruessner J, et al. In vivo coherent Raman imaging of the melanomagenesis-associated pigment pheomelanin. Sci Rep. 2016 Nov;6(1):37986

27 Wee E, Wolfe R, Mclean C, Kelly JW, Pan Y. Clinically amelanotic or hypomelanotic melanoma: anatomic distribution, risk factors, and survival. J Am Acad Dermatol. 2018 Oct; 79(4):645-651.e4.

28 Gong HZ, Zheng HY, Li J. Amelanotic melanoma. Melanoma Res. 2019 Jun;29(3):22130. 